\title{
Spectrum Monitoring Using SpectrumAnalysis LabVIEW Software, Nanoceptors, and Various Digitizing Solutions
}

\author{
by Joshua Smith
}

ARL-TR-7217

February 2015 


\section{NOTICES}

\section{Disclaimers}

The findings in this report are not to be construed as an official Department of the Army position unless so designated by other authorized documents.

Citation of manufacturer's or trade names does not constitute an official endorsement or approval of the use thereof.

Destroy this report when it is no longer needed. Do not return it to the originator. 


\title{
Army Research Laboratory
}

Adelphi, MD 20783-1138

ARL-TR-7217

February 2015

\section{Spectrum Monitoring Using SpectrumAnalysis LabVIEW Software, Nanoceptors, and Various Digitizing Solutions}

\author{
Joshua Smith
}

Sensors and Electron Devices Directorate, ARL 


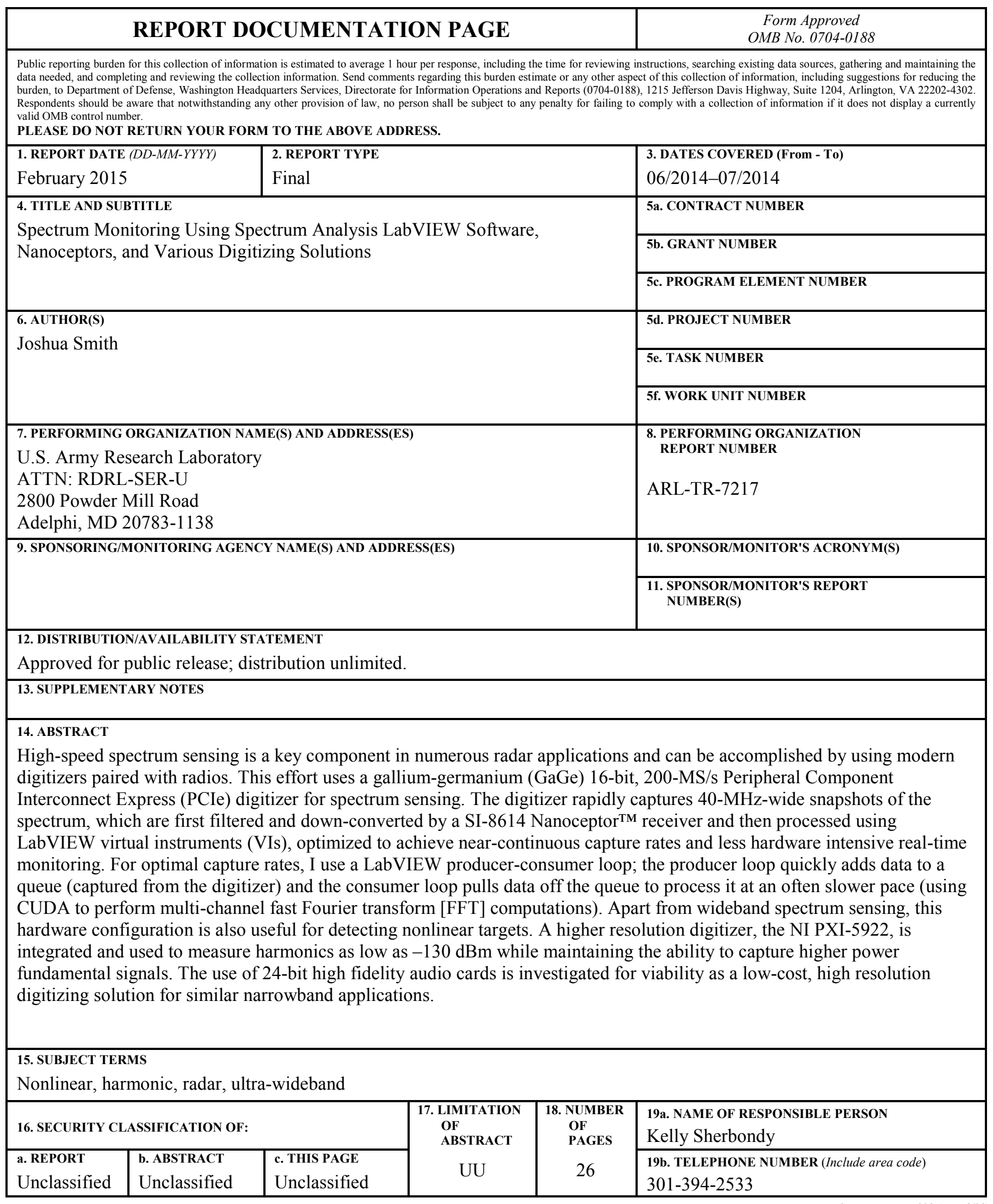




\section{Contents}

List of Figures $\quad$ v

List of Tables $\quad$ v

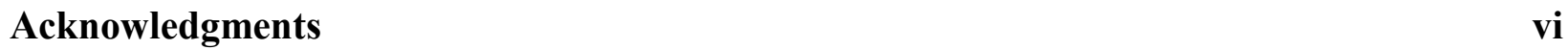

Student Bio

$\begin{array}{ll}\text { 1. Introduction/Background } & 1\end{array}$

2. External Hardware Configuration 1

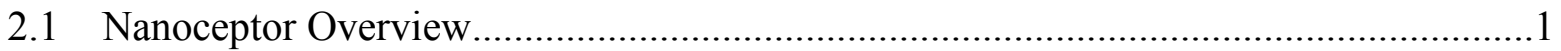

2.2 Basic Hardware Configuration ........................................................................

3. Hardware Initialization and Software Configuration 5

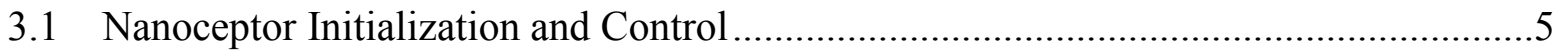

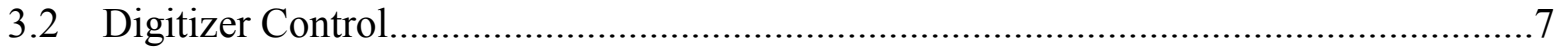

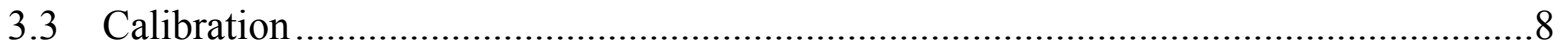

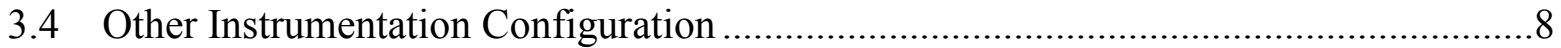

4. Software Usage $\quad 9$

4.1 External Instrument and Software Setup............................................................ 9

4.2 Harmonic Analysis ....................................................................................... 9

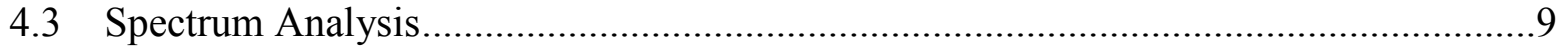

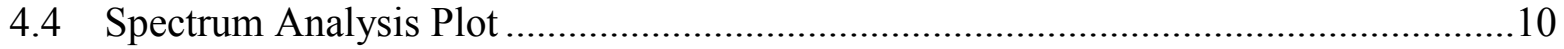

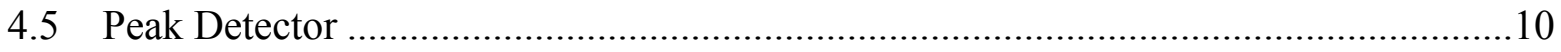

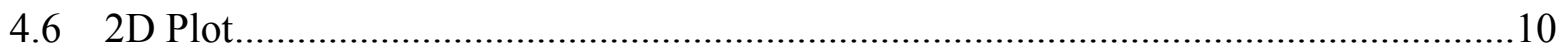

4.7 Hardware Diagram/Notes ............................................................................ 10

5. Nonlinear Characterization Hardware Configuration 10

6. Conclusion $\quad 11$ 
Appendix. Select Dependencies

List of Symbols, Abbreviations, and Acronyms

Distribution List

16 


\section{List of Figures}

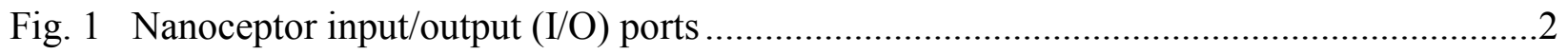

Fig. 2 Block diagram of the Nanoceptor front end ...........................................................

Fig. 3 Block diagram of the Nanoceptor filtering and switchable demodulation stages ..............3

Fig. 4 Example hardware configuration as depicted in the SpectrumAnalysis software..............4

Fig. 5 Nanoceptor port initialization ...............................................................................

Fig. 6 External clock reference configuration...................................................................

Fig. 7 Method for updating Nanoceptor tuned frequencies for calibration and sweeps ...............5

Fig. 8 Method for updating Nanoceptor tuning frequencies for manual frequency tuning or

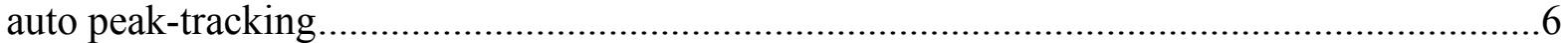

Fig. 9 Method for updating Nanoceptor tuning frequenciesfor scheduled harmonic analyses .....6

Fig. 10 Method for updating the Nanoceptor's switched IF parameters ..................................6

Fig. 11 Front panel interface for updating the Nanoceptor's switched IF parameters ................7

Fig. 12 High level block diagrams for data acquisition using various digitizers........................7

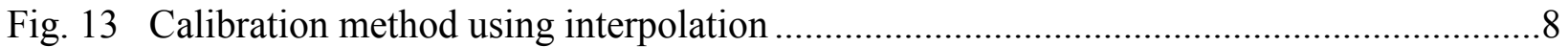

Fig. 14 Block diagram of a nonlinear hardware characterization bench setup .........................11

\section{List of Tables}

Table 1 Attenuation required to normalize signal amplitudes over installed Nanoceptor IF

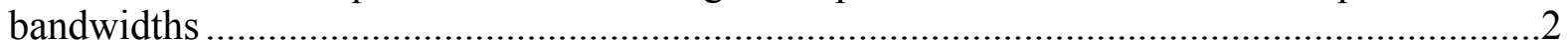

Table 2 Comparison of digitizer specifications ................................................................

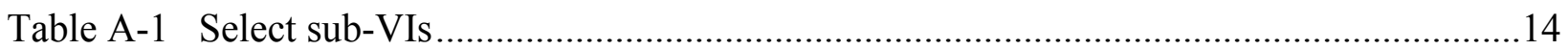




\section{Acknowledgments}

I appreciate the mentorship of Dr Anthony Martone and Mr Kelly Sherbondy. I would also like to thank Dr Andy Sullivan, Dr Matthew Higgins, Dr Gregory Mazzaro, Mr Marc Ressler, Mr Edward Viveiros, Dr Ronald Polcawich, Mr Brian Phelan, Mr Kyle Gallagher, and Mr Roger Cutitta for their assistance and support. I wish to acknowledge my fellow interns Richard Pooler, Kristopher Young, Jason Cornelius, and Philip Saponaro. 


\section{Student Bio}

Joshua Smith is an undergraduate student pursuing a BS degree in electrical engineering at the Pennsylvania State University, specializing in electromagnetics. He has experience designing and building electronic components for Penn State Racing, Penn State's Formula SAE team. 
INTENTIONALLY LEFT BLANK. 


\section{Introduction/Background}

Data acquisition systems are needed to monitor the radio frequency (RF) environment for a wide range of applications. One of these applications includes collecting data from the spectrum to analyze and develop techniques to mitigate RF interference. The traditional way to perform the necessary data collection is to use a spectrum analyzer, which takes a relatively long time to collect data. To alleviate this problem, a digitizing solution is implemented, which takes snapshots of time-domain data at various sampling rates. The Nyquist-Shannon sampling theorem dictates the maximum spectral bandwidth each sample rate can provide. For example, a digitizer with a sample rate of $200 \mathrm{MS} / \mathrm{s}$ has a maximum bandwidth of $100 \mathrm{MHz}$. By taking a large number of points per sample, digitizers capture a range of spectral data in a fraction of a second, whereas a spectrum analyzer requires a longer time, on the order of minutes, to capture the same sample.

The maximum frequency a digitizer detects is again limited by its maximum sample rate. In order to detect higher frequencies, a down-converter is required to mix the frequency range of interest to within the bandwidth limits of the digitizer. A receiver such as the SI-8614 Nanoceptor ${ }^{\mathrm{TM}}$ is used to automate the down-conversion process.

Data acquisition software called SpectrumAnalysis has been developed by LabVIEW to interface with Nanoceptors and digitizers. This report details how to use SpectrumAnalysis to collect, optimize, and analyze data for various RF investigations.

\section{External Hardware Configuration}

\subsection{Nanoceptor Overview}

The Nanoceptor very high frequency (VHF)/ultra high frequency (UHF) receiver simplifies frequency tuning up to $3 \mathrm{GHz}$ by removing the need for complex external mixing and filtering hardware. It has a series of programmatically switchable intermediate frequency (IF) filters and attenuators as well as other features such as AM, FM, LOG, and pulse demodulation. It is tunable from $20 \mathrm{MHz}$ to $3 \mathrm{GHz}$, in steps of $100 \mathrm{~Hz}$, to analyze a maximum signal of $+15 \mathrm{dBm}$ (without incurring damage). The tuned output is available from a wideband or a switched bandwidth 70-MHz IF output. The gain of the switched IF output is dictated by automatic gain control (AGC) and the IF bandwidth. When AGC is on, the amplitude of the signal is adjusted so that the highest gain is nominally $-30 \mathrm{dBm}$. Turning AGC off, the IF bandwidth dictates the approximate gain of the incoming signal. To normalize the gain per bandwidth, the receiver attenuation is adjusted as shown in Table 1. These approximate attenuation values were derived experimentally using a signal generator, which synthesized a $500 \mathrm{MHz},-50 \mathrm{dBm}$ signal to the 
input of the Nanoceptor. The attenuation for each IF bandwidth was adjusted until the measured amplitude of the 500-MHz signal matched that at the 40-MHz IF bandwidth. Measurements were taken with uncalibrated SpectrumAnalysis software using the NI PXI-5922 digitizer.

Table 1 Attenuation required to normalize signal amplitudes over installed Nanoceptor IF bandwidths

\begin{tabular}{|c|c|}
\hline IF Bandwidth & $\begin{array}{c}\text { Software Normalization Attenuation } \\
\text { (dB) }\end{array}$ \\
\hline $10 \mathrm{kHz}$ & 32 \\
\hline $30 \mathrm{kHz}$ & 28 \\
\hline $100 \mathrm{kHz}$ & 22 \\
\hline $400 \mathrm{kHz}$ & 13 \\
\hline $1.2 \mathrm{MHz}$ & 12 \\
\hline $5 \mathrm{MHz}$ & 6 \\
\hline $10 \mathrm{MHz}$ & 4 \\
\hline $25 \mathrm{MHz}$ & 0 \\
\hline $40 \mathrm{MHz}$ & 0 \\
\hline
\end{tabular}

Note: Enabling the Wideband Output toggle switch within SpectrumAnalysis disables all normalization attenuation.

Apart from 70-MHz wideband and switched IF outputs, the Nanoceptor also has video and phones outputs, as shown in Fig. 1, which are centered at the baseband. The video output provides controllable demodulation of the input signal, with selectable filters. The phones output provides an amplified version of the video output for headphone use.

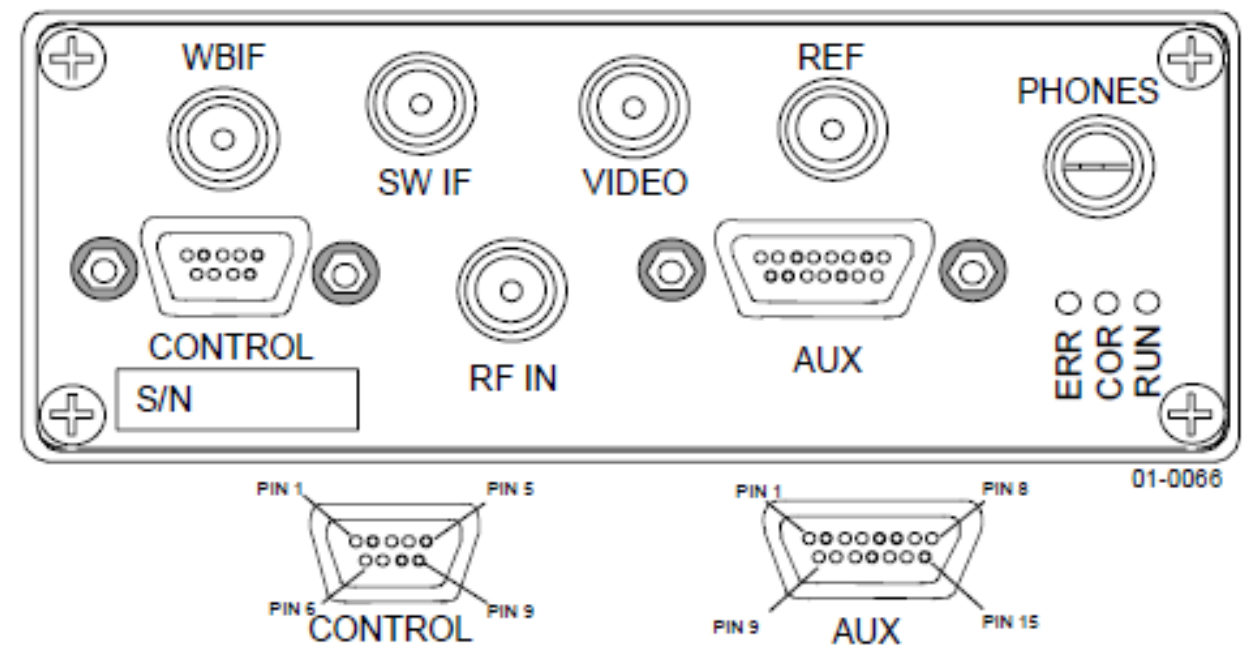

Fig. 1 Nanoceptor input/output (I/O) ports

The Nanoceptor has a REF port that either accepts a 10-MHz reference clock or outputs its own. When loading SpectrumAnalysis, the Nanoceptor is programmed to accept a 10-MHz reference. 
The Nanoceptor block diagram is shown in Figs. 2 and 3.

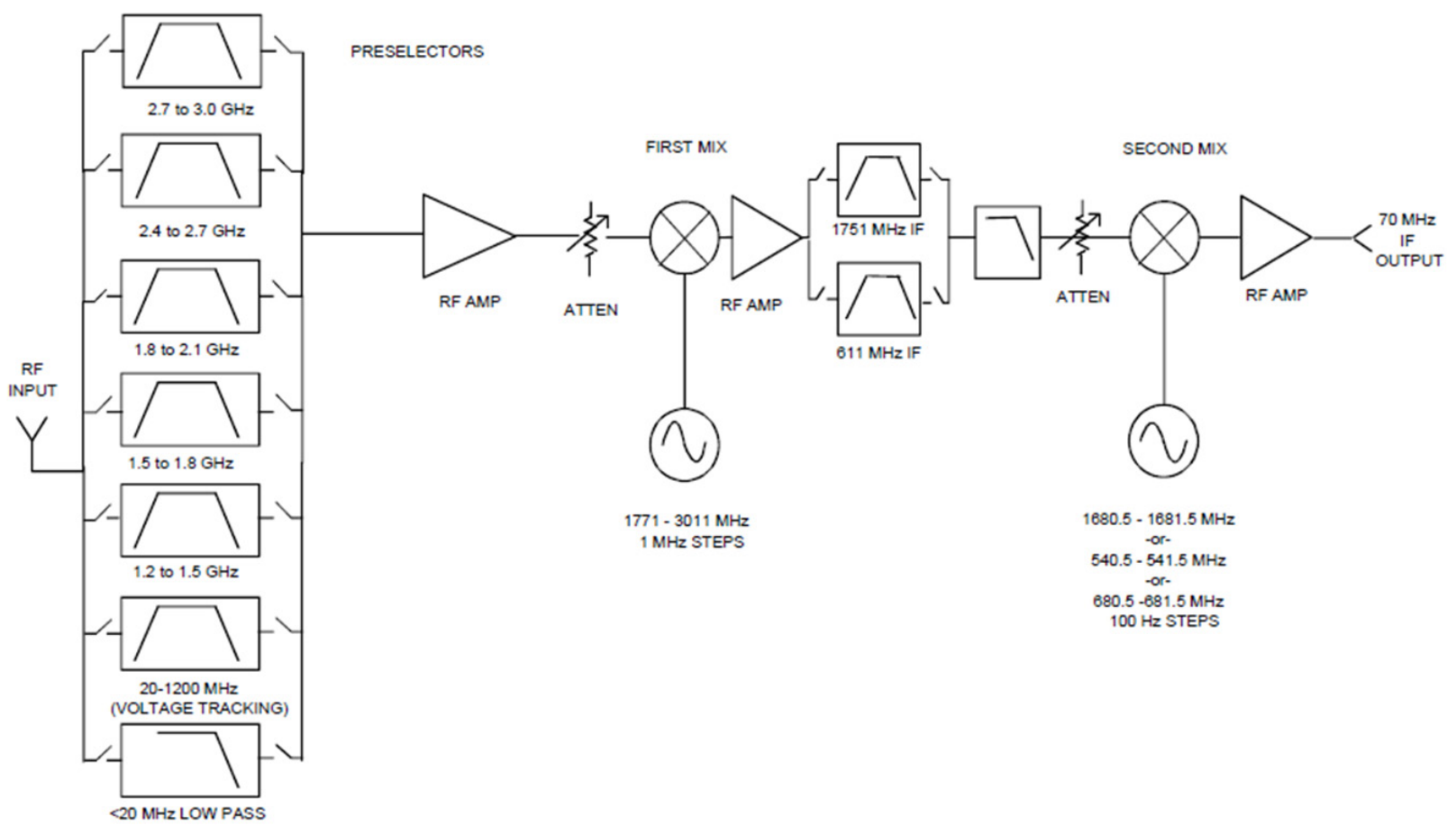

Fig. 2 Block diagram of the Nanoceptor front end

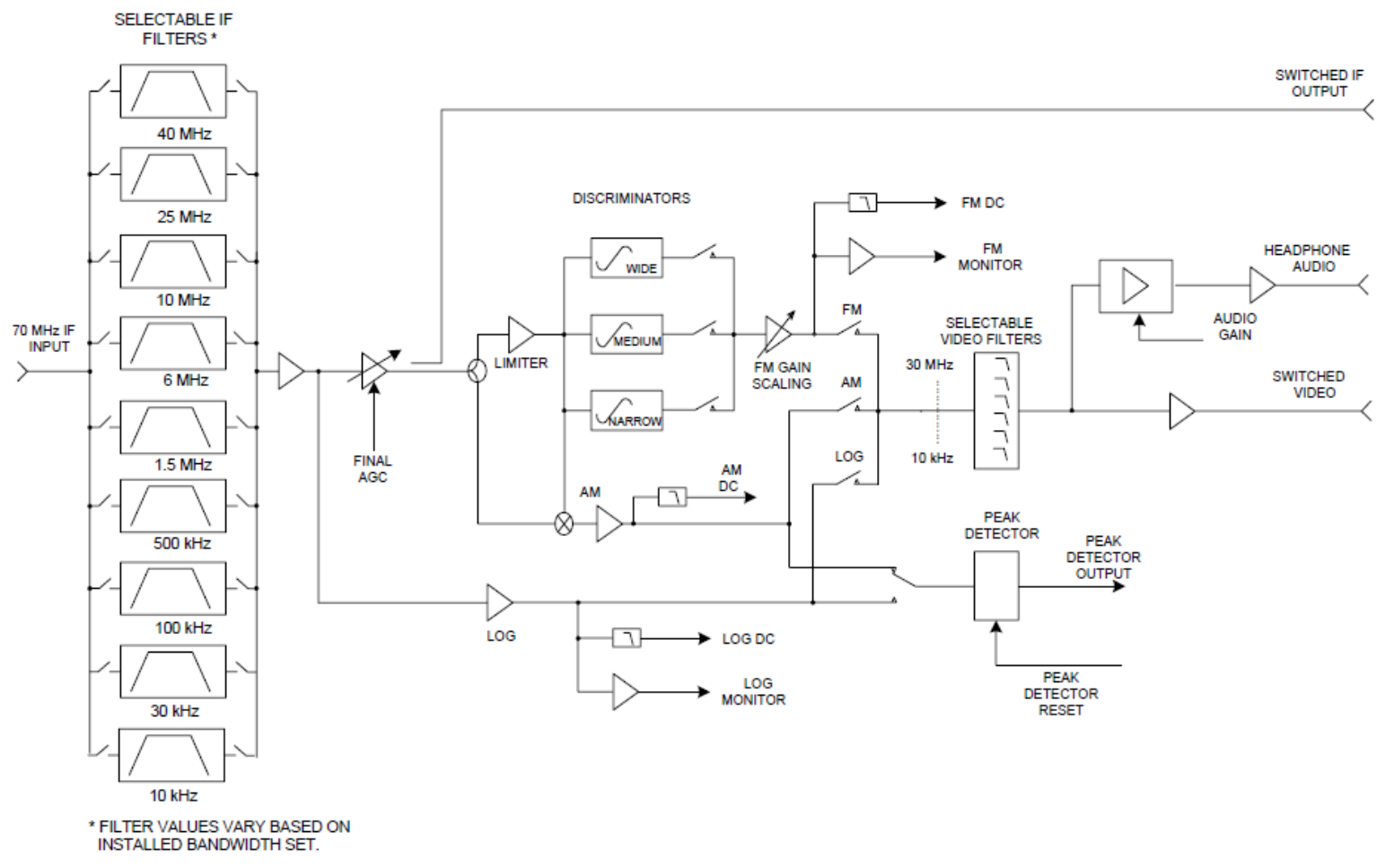

Fig. 3 Block diagram of the Nanoceptor filtering and switchable demodulation stages 


\subsection{Basic Hardware Configuration}

The SpectrumAnalysis software is designed to interface with either 1 or 2 Nanoceptors and at least 1 supported digitizer. The software requires these 2 different pieces of hardware and will not function without them. The currently supported digitizers and their specifications are listed in Table 2.

Table 2 Comparison of digitizer specifications

\begin{tabular}{c|ccc} 
Digitizer & Form Factor & Maximum Sample Rate & Bit Count \\
\hline GaGe CSE1622 & $\begin{array}{c}\text { Peripheral } \\
\text { Component } \\
\text { Interconnect Express } \\
\text { (PCIe) } x 16\end{array}$ & $200 \mathrm{MS} / \mathrm{s}$ & \\
NI PXI-5922 & PXIe & & \\
Creative Soundblaster ZxR & PCIe x16 & $96 \mathrm{kS} / \mathrm{s}$ (audio bitrate) & $16-24$ \\
Other Audio Device & -- & -- & 24
\end{tabular}

An example hardware configuration using the gallium-germanium (GaGe) CSE1622 and NI PXI-5922 digitizers is shown in Fig. 4. Figure 4 can be referenced from the "Hardware Diagram / Notes" tab of the SpectrumAnalysis software.

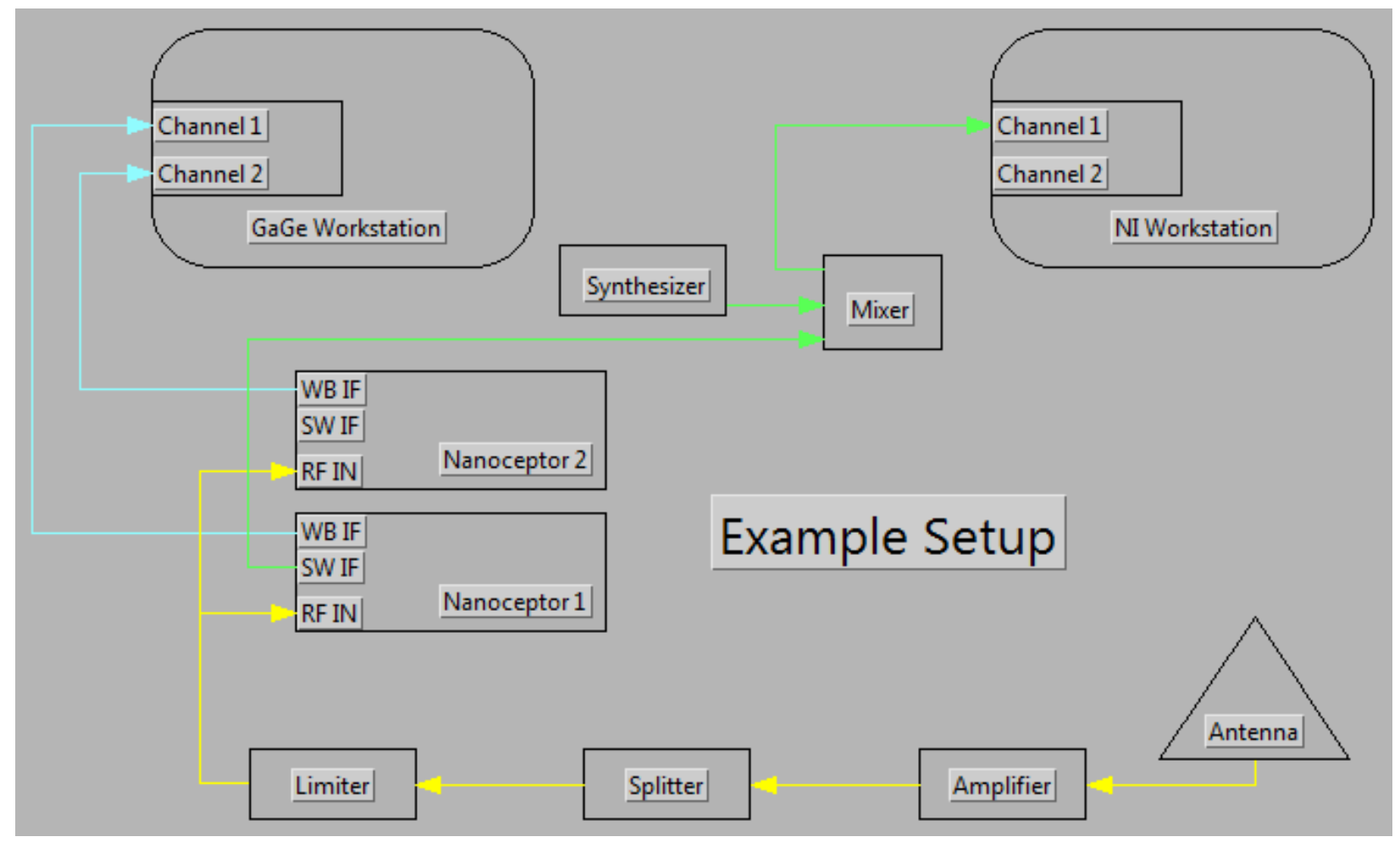

Fig. 4 Example hardware configuration as depicted in the SpectrumAnalysis software 


\section{Hardware Initialization and Software Configuration}

\subsection{Nanoceptor Initialization and Control}

The Nanoceptor COMM port is configured in the initialization stage of SpectrumAnalysis, as shown in Fig. 5. By default, the serial baud rate is 9600, but the Nanoceptor supports userconfigurable baud rates when a hardware switch is toggled.

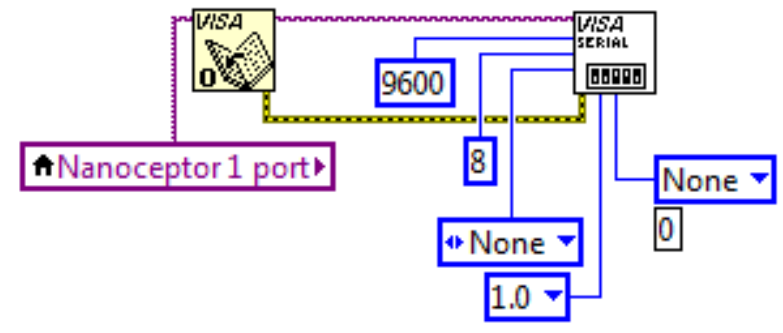

Fig. 5 Nanoceptor port initialization

The Nanoceptor is then set to accept an external reference by means of the SendNanoceptorCommand virtual instrument (VI), as shown in Fig. 6. It should be noted that the REF port will transmit a $10-\mathrm{MHz}$ reference signal until this command is sent.

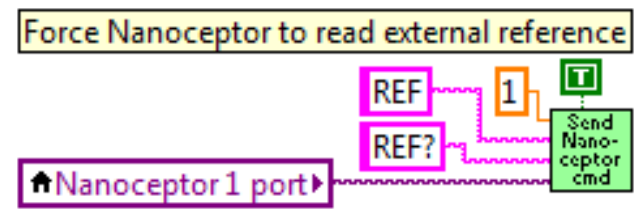

Fig. 6 External clock reference configuration

If the Nanoceptors are scheduled to sweep frequencies for routine tasks such as spectrum analysis and calibration, they are tuned to the first frequency in the initialization stage of SpectrumAnalysis. Any subsequent scheduled updates to the tuned frequencies are programmed in the main loop, as shown in Fig. 7.

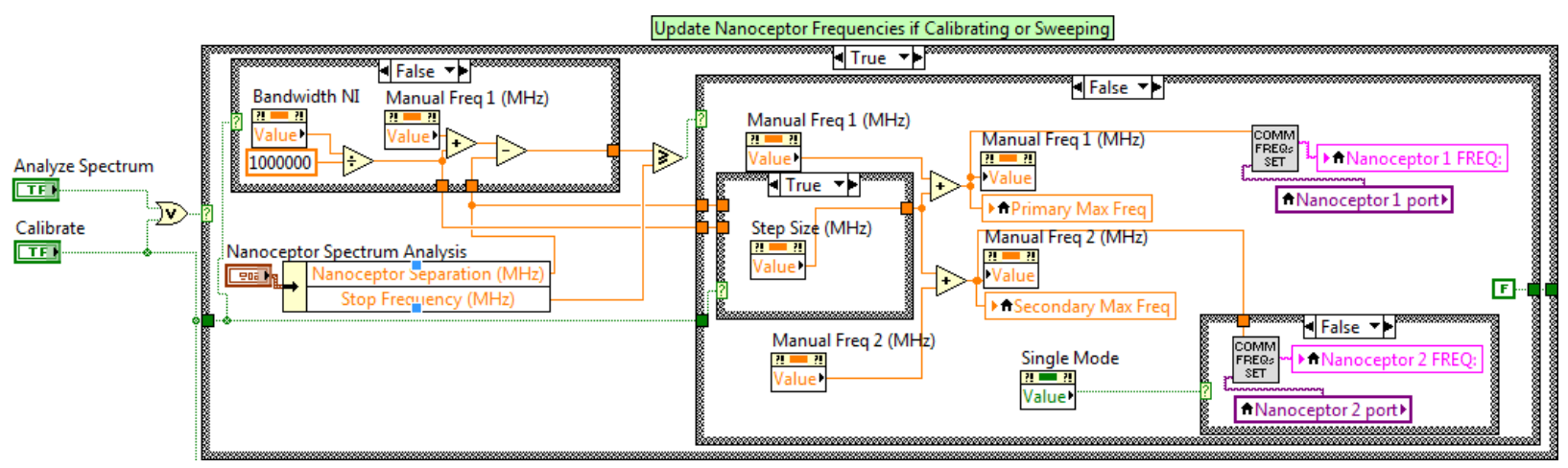

Fig. 7 Method for updating Nanoceptor tuned frequencies for calibration and sweeps 
If the user manually changes the tuning frequency, or if Auto-Tracking is enabled, the frequencies are updated using the method shown in Fig. 8.

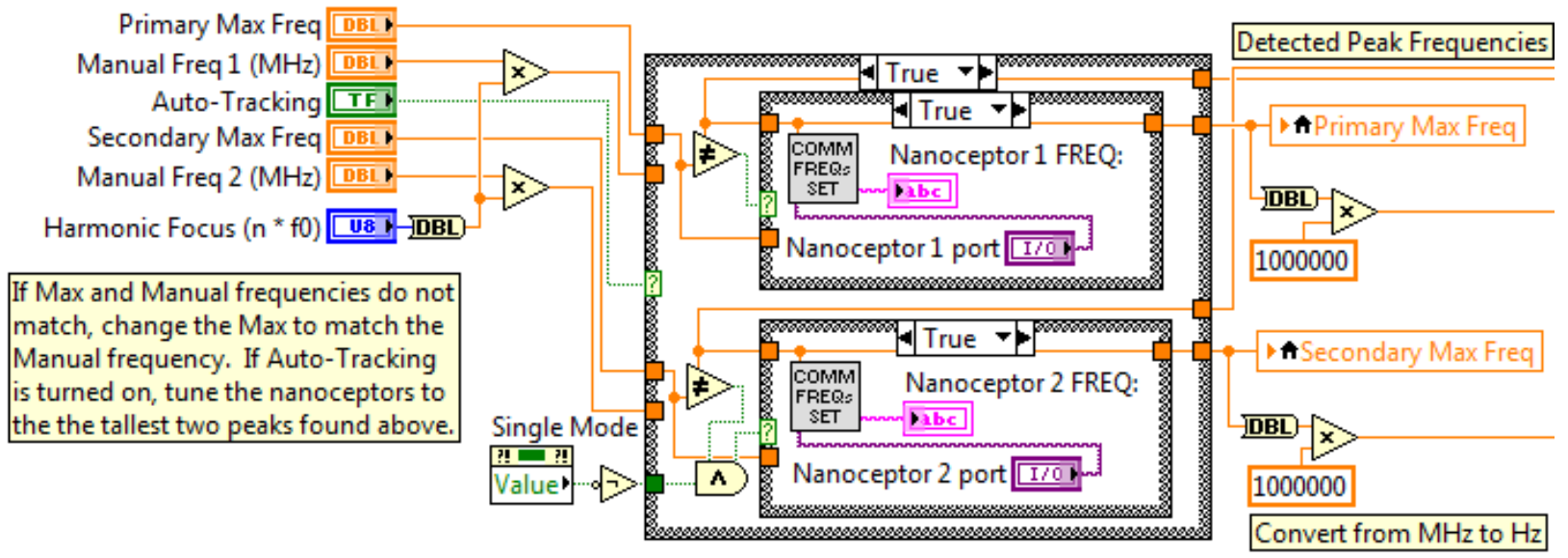

Fig. 8 Method for updating Nanoceptor tuning frequencies for manual frequency tuning or auto peak-tracking

The Nanoceptor tuning frequencies are also updated if a harmonic analysis is scheduled (Fig. 9).

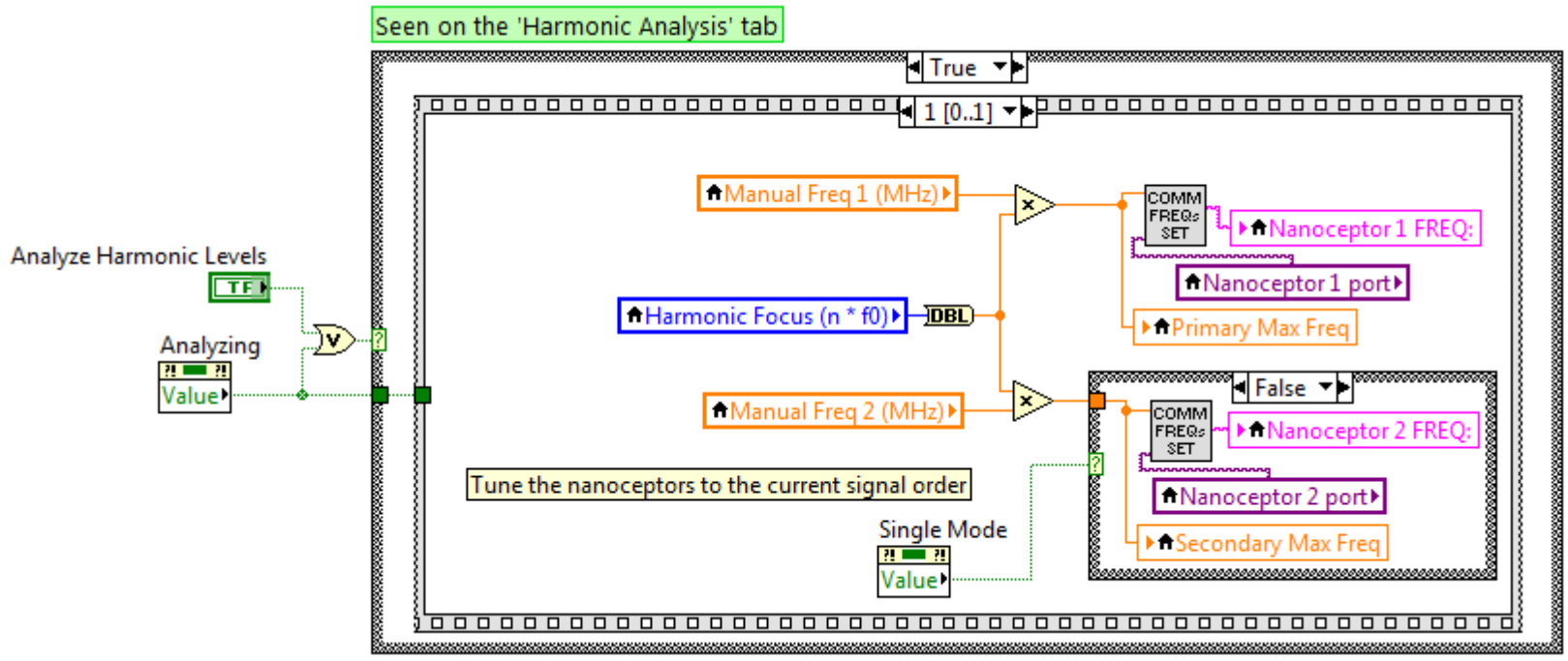

Fig. 9 Method for updating Nanoceptor tuning frequenciesfor scheduled harmonic analyses

The switched IF bandwidth filters are controlled via the top of the front panel (Fig. 10). The parameters are loaded into the Nanoceptor when the Set button is pressed, as shown in Fig. 11.

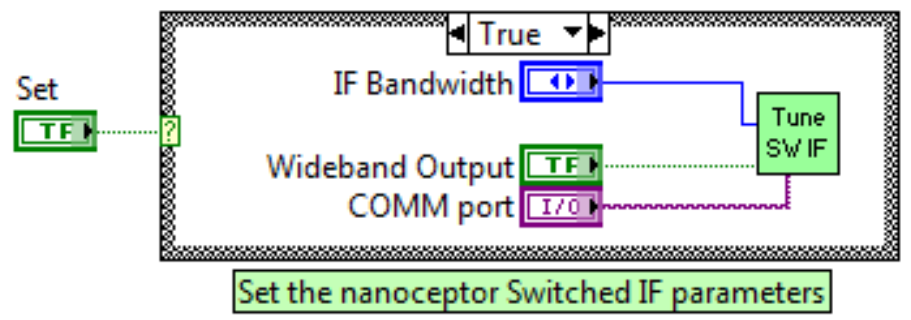

Fig. 10 Method for updating the Nanoceptor's switched IF parameters 


\begin{tabular}{|c|c|c|c|c|}
\hline Wideband Output & IF Bandwidth & COMM p & & \\
\hline (3) Narrowband Output & $40 \mathrm{MHz}$ & $\mathrm{I} / \mathrm{COM} 3$ & $\checkmark$ & E \\
\hline
\end{tabular}

Fig. 11 Front panel interface for updating the Nanoceptor's switched IF parameters

To initialize the Nanoceptors on the front panel, simply select their respective ports before starting or unpausing the software.

\subsection{Digitizer Control}

SpectrumAnalysis is built upon a GaGe example VI called GaGeAcquire, and thus follows its layout and programming structure. Numerous frames of the sequence are dedicated to initializing and shutting down the GaGe digitizer. In Frame 4 of the main loop, the time-domain data are recorded from the digitizer(s) that are selected. Figure 12 displays the data acquisition process for a few digitizing options.

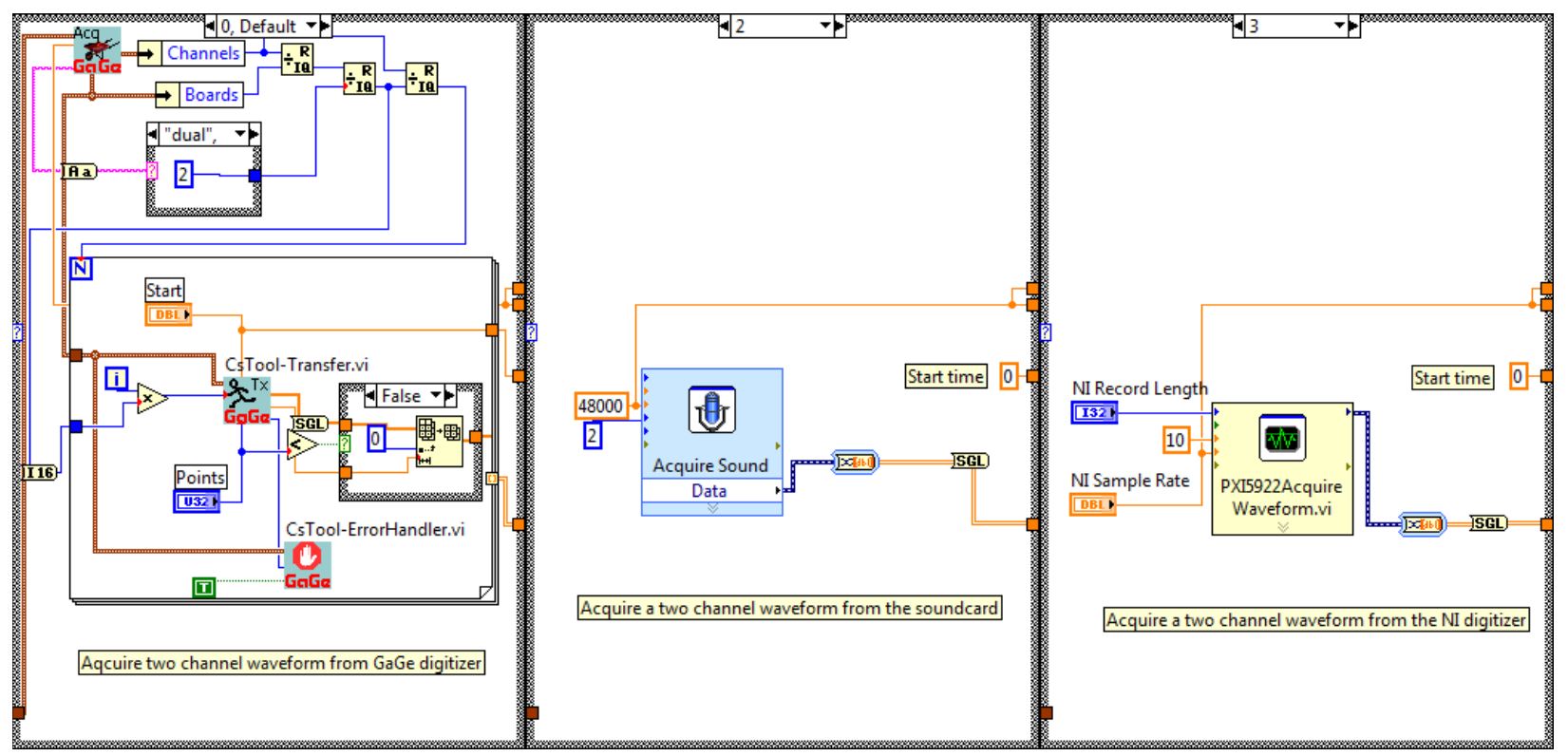

Fig. 12 High level block diagrams for data acquisition using various digitizers

The digitizers are configurable via the front panel. The record length (number of points taken per capture) and sample rate are used to configure the digitizing hardware, while the IF and bandwidth are used to configure the graphs and spectral data. The latter permits the software to trace back through the mixing stages in order to determine the true frequency of the signal. These parameters are set in the "GaGe Setup" and "External Instrument and Software Setup" tabs on the front panel. 


\subsection{Calibration}

SpectrumAnalysis software uses NVIDIA CUDA technology to perform the fast Fourier transforms (FFTs) by means of faster parallel processing. After performing the FFT, the spectral data must be scaled to be converted to the spectral power density, as shown in Eq. 1:

$$
\text { Spectral Power Density }(d B)=10 * \log \left(\frac{|X|^{2}}{N / 2}\right) \text {, }
$$

where $\mathrm{X}$ is the raw FFT output and $\mathrm{N}$ is the number of time samples used in the FFT.

The spectral power density then has to be shifted along the amplitude axis to accurately measure absolute power. Because the signal traverses through various pieces of hardware before reaching the digitizer, the power of the signal at the front end of the digitizer is likely not to match the power produced by the signal source anyway. Instead of spending more computation time converting the spectral power density to absolute power, a calibration function was implemented to calibrate out all software and hardware gain offsets.

There are two main parts in the calibration process: saving a calibration file and using a previously saved calibration to adjust the signal. To save a calibration file, the Nanoceptors and an external signal generator are scheduled to sweep the same frequencies at the same time. The software finds the spectral amplitude of the incoming signal and compares it to what the signal generator is sourcing. The difference is then appended to a calibration file along with frequency information. To use that file for calibrating the incoming signal, the interpolated calibration values are added to the spectral signal, as shown in Fig. 13.

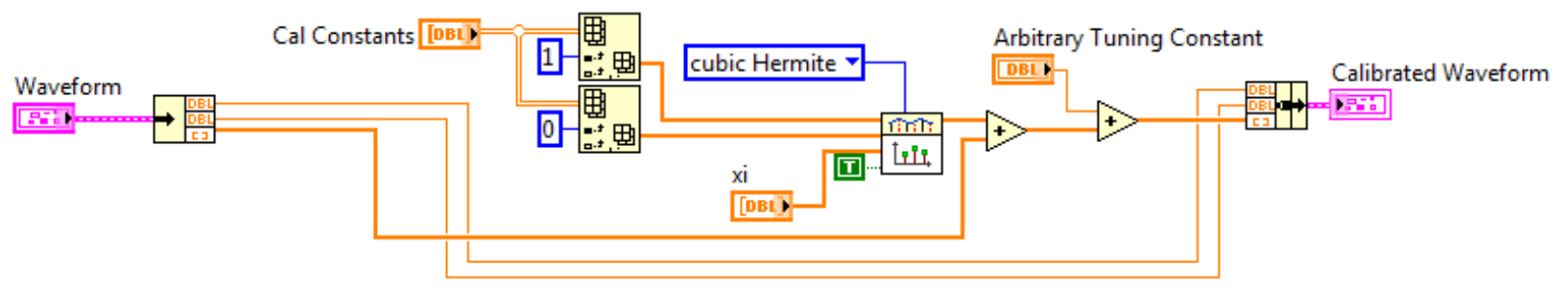

Fig. 13 Calibration method using interpolation

Instructions for performing and loading a calibration are found on the "External Instrument and Software Setup" tab of the SpectrumAnalysis software.

\subsection{Other Instrumentation Configuration}

To use other instrumentation, for example, the oscilloscope, simply select the resource from its respective list and select the "Use X" button, where $X$ is the instrument name. 


\section{Software Usage}

When SpectrumAnalysis is loaded, it is initialized to a paused state. The user can change all software configurations and hardware settings before the software reads them. The tutorial pane on the "External Instrument and Software Setup" tab can be referenced for assistance with tasks such as calibration, spectrum analysis, and noise reduction. Hardware function can be verified in the "GaGe Setup" tab, where there are raw-time and frequency-domain graphs of both channels, without any extra processing. The rest of this section analyzes a few functions of the "External Instrument and Software Setup" tab and the complete function of other tabs.

\subsection{External Instrument and Software Setup}

To capture data as fast as possible and process the data at the computer's pace, "Queue Size" should be set to a higher number (e.g., 5000). To capture the data at the same rate as it is processed and displayed, the queue size should be set to 1 . To use 2 Nanoceptors, ensure that "Single Mode" is deselected. This must be selected if there is only 1 Nanoceptor connected to the host. When loading a calibration or performing a sweep, the software must be newly started.

\subsection{Harmonic Analysis}

The "Harmonic Analysis" tab is used to rapidly measure the power level of signals at commonly analyzed frequencies and currently has 2 parts. The left side of the tab quickly analyzes the first nth-order terms of the fundamental frequency, from either the Channel 1 or Channel 2 inputs. The right side of the tab quickly measures the amplitudes of intermodulation products at any frequency. The user first sets the center frequency, which, for instance, can be the frequency of the second-order term for nonlinear applications. The user then sets the amount of intermodulation products to analyze on either side of the center frequency, as well as the spacing in $\mathrm{Hz}$ between each product. When the "Measure Intermods" button is pressed, the frequency and amplitude of each intermodulation product appears in the "Intermod Values" indicator.

\subsection{Spectrum Analysis}

This tab displays the processed spectral waveforms of Channels 1 and 2. Depending on the amount of mixing stages used before the digitizer, the waveforms may need to be flipped side to side. A known signal is used to determine if the graphs are oriented properly by either analyzing the shape of the signal, sweeping the signal, and tracking its direction of movement; or changing the tuning frequency of the Nanoceptor and tracking how the signal moves with respect to that.

To better analyze a signal or see very low signals, an averaging function is used. To enable averaging, simply set the amount of iterations and select the "Averaging" button. The button should be deselected when changing frequencies for optimal performance. 


\subsection{Spectrum Analysis Plot}

If a spectrum analysis was recorded and saved to a file, the plot appears on this page for rapid reviewing.

\subsection{Peak Detector}

This tab interfaces with the FFT channel of an oscilloscope to find spectral peaks. If AutoTracking is enabled, the Nanoceptors are automatically tuned to the tallest peaks. "Peak Detector Width" specifies the number of surrounding points that each peak must be taller than to be considered valid. This value must be greater than 3 , yet smaller than the widths of the peaks of interest. Thus, 5 is generally a good width for detecting real peaks. "Side Padding" specifies the amount of points on either side of the graph to ignore when peak detecting. This is useful for ignoring higher DC gain. The software finds the average tallest peaks over the previous $\mathrm{N}$ iterations, where $\mathrm{N}$ is specified as the "Detection Accumulation Size." The red threshold bar on the graph sets the minimum amplitude of a point for a Nanoceptor tuning candidate. Detected peaks are labeled in red.

\subsection{D Plot}

This tab shows an intensity chart of the signal on Channel 1. Note that the axes are labeled Frequency vs. Time. This is useful for analyzing the changing signal over time. The amplitude color scheme can be adjusted to better analyze any range of values.

\subsection{Hardware Diagram/Notes}

An example hardware diagram for spectrum analysis can be referenced from this tab.

\section{Nonlinear Characterization Hardware Configuration}

Figure 14 is a rough block diagram of a functional nonlinear hardware characterization bench setup. The chip-scale atomic clock (CSAC) provides a 10-MHz reference clock to the SignalCore Synthesizer. This clock is also used to synchronize the Nanoceptor and the digitizer. The SignalCore Synthesizer sets a reference frequency for the AD9914 Synthesizer to create a 2-tone signal about. This 2-tone signal is then injected into a device under test (DUT), and the reflection is coupled into the Nanoceptor through a bandpass filter and receive chain. The Nanoceptor filters and mixes the reflected signal down to a $70-\mathrm{MHz}$ IF. The $70-\mathrm{MHz}$ output is externally mixed to a lower frequency, within the bandwidth of the digitizer used. The digitized signal is then processed using a computer with SpectrumAnalysis software. 


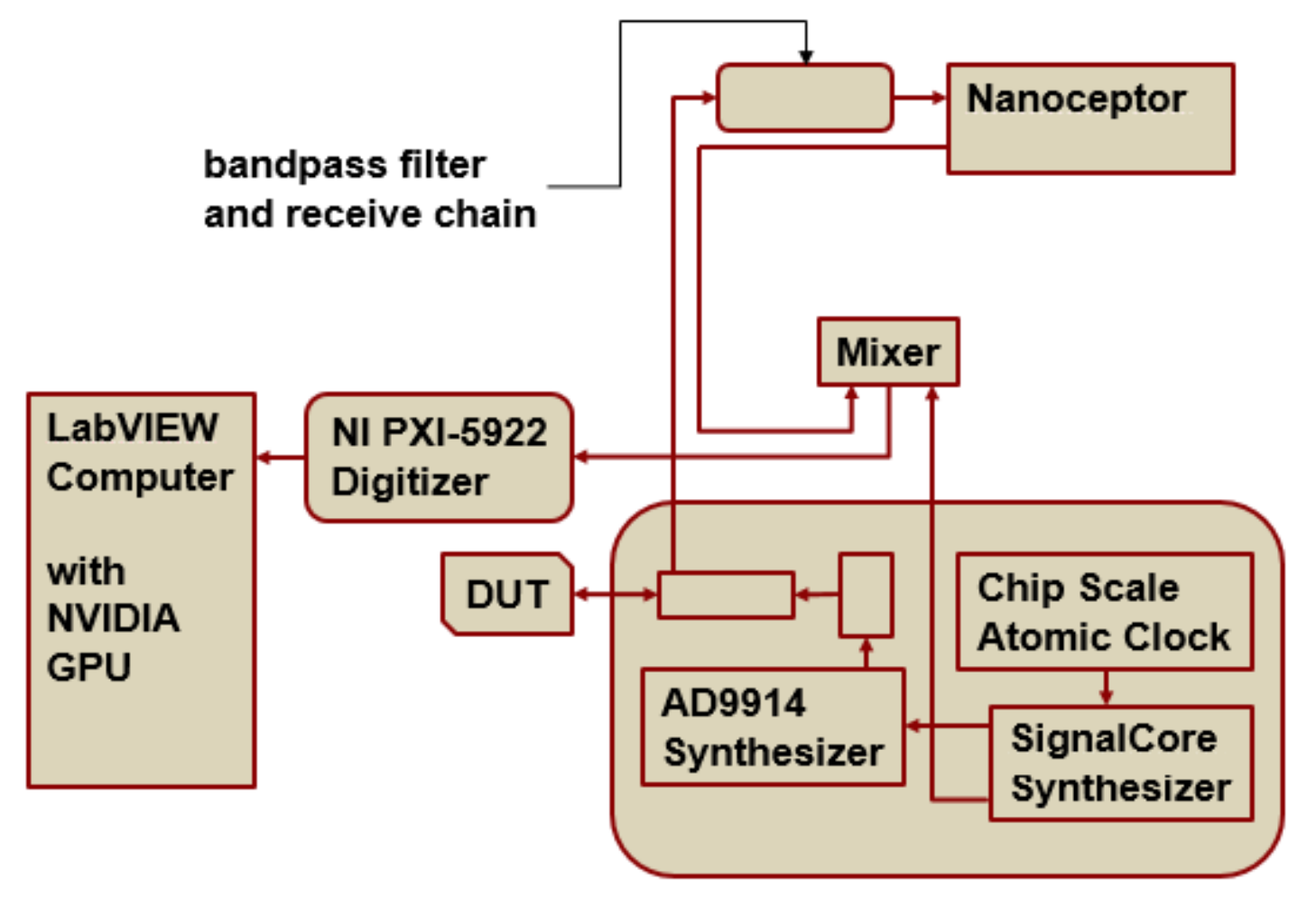

Fig. 14 Block diagram of a nonlinear hardware characterization bench setup

\section{Conclusion}

A key part of this project was implementing a fully functional software-defined spectrum analysis capability that incorporates software calibration, basic hardware control, Nanocepter control with various visualization controls, and output of harmonic- and spectrum-derived data via LabVIEW. Using the SpectrumAnalysis VI software that I created, one can collect, optimize, and analyze data for various RF investigations using the Nanocepter hardware and digitizers. This SpectrumAnalysis VI is currently being used as part of the US Army Research Laboratory's Sensors and Electron Devices Directorate (ARL-SEDD), RF Signal Processing and Modeling Branch's Sensitive RF (SeRF) Team's RF Enabled Detection Location and IED Neutralization Evaluation (REDLINE) Project for harmonic radar characterization. 
INTENTIONALLY LEFT BLANK. 
Appendix. Select Dependencies 
Table A-1 lists a selection of the sub-VIs used in the SpectrumAnalysis software.

Table A-1 Select sub-VIs

\begin{tabular}{|c|c|c|}
\hline 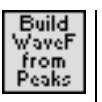 & BuildWaveformFromPeaks.vi & Converts the detected peaks to a waveform of points \\
\hline \begin{tabular}{|c||} 
CAL \\
WAVE- \\
FORM \\
\end{tabular} & CalibrateWaveform.vi & $\begin{array}{l}\text { Uses calibration constants to dynamically calibrate a waveform. } \\
\text { Requires GetXValuesFromWaveform.vi }\end{array}$ \\
\hline \begin{tabular}{|c|} 
Center \\
Freq \\
Finder \\
\end{tabular} & CenterFrequencyFinder.vi & Detects the highest peak about the tuning frequency \\
\hline \begin{tabular}{|l|} 
IIGHEST \\
PFEAKS \\
hite \\
\end{tabular} & FindHighestXPeaks.vi & Detects and reports the highest $\mathrm{X}$ peaks of a waveform \\
\hline $\begin{array}{l}\text { Gett } \\
\text { Peoki ot } \\
\text { het } \\
\end{array}$ & GetPeakCloseToValue.vi & $\begin{array}{l}\text { Finds the peak at a certain frequency, within a threshold of } \\
\text { surrounding points }\end{array}$ \\
\hline \begin{tabular}{|c|} 
GET: \\
VALUES
\end{tabular} & GetXValuesFromWaveform.vi & Returns an array of the frequencies at each point \\
\hline \begin{tabular}{|c|} 
Resod \\
Groph \\
hene \\
\end{tabular} & GraphSpectrumData.vi & Reads and displays a spectrum graph from a file \\
\hline $\begin{array}{l}\text { Intermod } \\
\text { Vislues } \\
\text { then }\end{array}$ & IntermodValueFinder.vi & Returns the intermodulation products about a certain frequency \\
\hline \begin{tabular}{|l|} 
Nano- \\
ceptor \\
Giraph
\end{tabular} & NanoceptorGraph.vi & Processes the raw signal data into a corrected waveform \\
\hline $\begin{array}{l}\text { Tune } \\
\text { SW' IF }\end{array}$ & NanoceptorTuneSWIF.vi & Programs the SW IF output of the Nanoceptor \\
\hline $\begin{array}{c}\text { Pesk } \\
C \\
C\end{array}$ & PeakCounter.vi & Tracks the average tallest peaks of a waveform \\
\hline 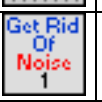 & ReduceNoise1.vi & Reduces noise using method 1 \\
\hline \begin{tabular}{|c|}
$\begin{array}{c}\text { Get Bid } \\
\text { of } \\
\text { Noige } \\
2\end{array}$ \\
\end{tabular} & ReduceNoise2.vi & Reduces noise using method 2 \\
\hline \begin{tabular}{|c|} 
Send \\
Nono- \\
ceptor \\
cmd \\
\end{tabular} & SendNanoceptorCommand.vi & Wrapper for simple_communication_vi.vi \\
\hline \begin{tabular}{|c|} 
COMM \\
FREQE \\
SET
\end{tabular} & SetNanoceptorFRQs.vi & Rapidly changes the Nanoceptor tuning frequency \\
\hline \begin{tabular}{l|l|} 
rimple \\
comM
\end{tabular} & simple_communication_vi.vi & Programs the Nanoceptor and queries a response \\
\hline Averstrum & SpectrumAveraging.vi & Averages a waveform over the previous $\mathrm{N}$ iterations \\
\hline \begin{tabular}{|c|} 
Get \\
Wove- \\
form \\
Center \\
\end{tabular} & TakeCenterOfWaveform.vi & $\begin{array}{l}\text { Finds tallest peak in single tone waveform (used as the center } \\
\text { frequency for a calibration) }\end{array}$ \\
\hline \begin{tabular}{|c|c|} 
CAL \\
0010 \\
0010 \\
$\rightarrow$ \\
\end{tabular} & WriteCalFile.vi & Iteratively saves a calibration file \\
\hline 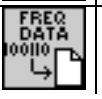 & WriteFrequencyDomainData.vi & Iteratively saves a spectrum analysis file \\
\hline
\end{tabular}




\section{List of Symbols, Abbreviations, and Acronyms}

$\mathrm{AGC}$

ARL

CSAC

DUT

FFT

$\mathrm{GaGe}$

IF

REDLINE

RF

SEDD

SeRF

UHF

VHF

VI automatic gain control

US Army Research Laboratory

chip-scale atomic clock

device under test

fast Fourier transform

gallium-germanium

intermediate frequency

RF Enabled Detection Location and IED Neutralization Evaluation

radio frequency

Sensors and Electron Devices Directorate

Sensitive RF

ultra high frequency

very high frequency

virtual instrument 


$\begin{array}{cl}1 & \text { DEFENSE TECH INFO CTR } \\ (\text { PDF }) & \text { ATTN DTIC OCA } \\ 1 & \text { GOVT PRINTG OFC } \\ (\mathrm{PDF}) & \text { A MALHOTRA } \\ 2 & \text { US ARMY RSRCH LABORATORY } \\ (\mathrm{PDF}) & \text { ATTN IMAL HRA MAIL \& RECORDS MGMT } \\ & \text { ATTN RDRL CIO LL TECHL LIB } \\ 5 & \text { US ARMY RSRCH LAB } \\ (\text { PDF }) & \text { ATTN RDRL SER U K SHERBONDY } \\ & \text { ATTN RDRL SER U M HIGGINS } \\ & \text { ATTN RDRL SER U T MARTONE } \\ & \text { ATTN RDRL SER U M RESSLER } \\ & \text { ATTN RDRL SER U D MCNAMARA }\end{array}$

